\title{
New Extension of Unified Family of Apostol-Type Polynomials and Numbers
}

\author{
Beih El-Sayed El-Desouky, Rabab Sabry Gomaa \\ Department of Mathematics, Mansoura University, Mansoura, Egypt \\ Email: b_desouky@yahoo.com, dr.rsg12@yahoo.com
}

Received 22 May 2015; accepted 1 August 2015; published 5 August 2015

Copyright (C) 2015 by authors and Scientific Research Publishing Inc.

This work is licensed under the Creative Commons Attribution International License (CC BY).

http://creativecommons.org/licenses/by/4.0/

(c) (i) 0pen Access

\section{Abstract}

The purpose of this paper is to introduce and investigate new unification of unified family of Apostol-type polynomials and numbers based on results given in [1] [2]. Also, we derive some properties for these polynomials and obtain some relationships between the Jacobi polynomials, Laguerre polynomials, Hermite polynomials, Stirling numbers and some other types of generalized polynomials.

\section{Keywords}

Euler, Bernoulli and Genocchi Polynomials, Stirling Numbers, Laguerre Polynomials, Hermite Polynomials

\section{Introduction}

The generalized Bernoulli polynomials $B_{n}^{(\alpha)}(x)$ of order $\alpha \in \mathbb{C}$ and the generalized Euler polynomials are defined by (see [3]):

$$
\left(\frac{t}{\mathrm{e}^{t}-1}\right)^{\alpha} \mathrm{e}^{\mathrm{xt}}=\sum_{n=0}^{\infty} B_{n}^{(\alpha)}(x) \frac{t^{n}}{n !},\left(|t|<2 \pi ; 1^{\alpha}:=1\right)
$$

and

$$
\left(\frac{t}{\mathrm{e}^{t}+1}\right)^{\alpha} \mathrm{e}^{x t}=\sum_{n=0}^{\infty} E_{n}^{(\alpha)}(x) \frac{t^{n}}{n !},\left(|t|<\pi ; 1^{\alpha}:=1\right),
$$

where $\mathbb{C}$ denotes the set of complex numbers.

Recently, Luo and Srivastava [4] introduced the generalized Apostol-Bernoulli polynomials $B_{n}^{(\alpha)}(x ; \lambda)$ and the generalized Apostol-Euler polynomials $E_{n}^{(\alpha)}(x ; \lambda)$ as follows. 
Definition 1.1. (Luo and Srivastava [4]) The generalized Apostol-Bernoulli polynomials $B_{n}^{(\alpha)}(x ; \lambda)$ of order $\alpha \in \mathbb{C}$ are defined by the generating function

$$
\begin{aligned}
& \left(\frac{t}{\lambda \mathrm{e}^{t}-1}\right)^{\alpha} \mathrm{e}^{x t}=\sum_{n=0}^{\infty} B_{n}^{(\alpha)}(x ; \lambda) \frac{t^{n}}{n !} \\
& \left(|t|<2 \pi \text { when } \lambda=1 ;|t|<|\log \lambda| \text {, when } \lambda \neq 1 ; 1^{\alpha}:=1\right) .
\end{aligned}
$$

Definition 1.2. (Luo [5]) The generalized Apostol-Euler polynomials $E_{n}^{(\alpha)}(x ; \lambda)$ of order $\alpha \in \mathbb{C}$ are defined by the generating function

$$
\begin{aligned}
& \left(\frac{t}{\lambda \mathrm{e}^{t}+1}\right)^{\alpha} \mathrm{e}^{x t}=\sum_{n=0}^{\infty} E_{n}^{(\alpha)}(x ; \lambda) \frac{t^{n}}{n !} \\
& \left(|t|<\pi \text { when } \lambda=1 ;|t|<|\log (-\lambda)| \text {, when } \lambda \neq 1 ; 1^{\alpha}:=1\right) .
\end{aligned}
$$

Natalini and Bernardini [6] defined the new generalization of Bernoulli polynomials in the following definition.

Definition 1.3. The generalized Bernoulli polynomials $B_{n}^{[m-1]}(x), m \in \mathbb{N}$, are defined, in a suitable neighbourhood of $t=0$ by means of generating function

$$
\frac{t^{m} \mathrm{e}^{x t}}{\mathrm{e}^{t}-\sum_{l=0}^{m-1} \frac{t^{l}}{l !}}=\sum_{n=0}^{\infty} B_{n}^{[m-1]}(x) \frac{t^{n}}{n !} .
$$

Recently, Tremblay et al. [7] investigated a new class of generalized Apostol-Bernoulli polynomial as follows.

Definition 1.4. The generalized Apostol-Bernoulli polynomials $B_{n}^{[m-1, \alpha]}(x ; \lambda)$ of order $\alpha \in \mathbb{C}, m \in \mathbb{N}$, are defined, in a suitable neighbourhood of $t=0$ by means of generating function

$$
\left(\frac{t^{m}}{\lambda \mathrm{e}^{t}-\sum_{l=0}^{m-1} \frac{t^{l}}{l !}}\right)^{\alpha} \mathrm{e}^{x t}=\sum_{n=0}^{\infty} B_{n}^{[m-1, \alpha]}(x ; \lambda) \frac{t^{n}}{n !} .
$$

Also, Sirvastava et al. [1] introduced a new interesting class of Apostol-Bernoulli polynomials that are closely related to the new class that we present in this paper. They investigated the following form.

Definition 1.5. Let $a, b, c \in \mathbb{R}^{+}(a \neq b)$ and $n \in \mathbb{N}_{0}$. Then the generalized Bernoulli polynomials $\mathfrak{B}_{n}^{(\alpha)}(x ; \lambda ; a, b, c)$ of order $\alpha \in \mathbb{C}$ are defined by the following generating function:

$$
\begin{aligned}
& \left(\frac{t}{\lambda b^{t}-a^{t}}\right)^{\alpha} c^{x t}=\sum_{n=0}^{\infty} \mathfrak{B}_{n}^{(\alpha)}(x ; \lambda ; a, b, c) \frac{t^{n}}{n !} \\
& \left(\left|t \log \left(\frac{a}{b}\right)\right|<|\log \lambda| ; 1^{\alpha}:=1\right) .
\end{aligned}
$$

This sequel to the work by Sirvastava et al. [2] introduced and investigated a similar generalization of the family of Euler polynomials defined as follows.

Definition 1.6. Let $a, b, c \in \mathbb{R}^{+}(a \neq b)$ and $n \in \mathbb{N}_{0}$. Then the generalized Euler polynomials $\mathfrak{E}_{n}^{(\alpha)}(x ; \lambda ; a, b, c)$ of order $\alpha \in \mathbb{C}$ are defined by the following generating function

$$
\begin{aligned}
& \left(\frac{t}{\lambda b^{t}+a^{t}}\right)^{\alpha} c^{x t}=\sum_{n=0}^{\infty} \mathfrak{E}_{n}^{(\alpha)}(x ; \lambda ; a, b, c) \frac{t^{n}}{n !} \\
& \left(\left|t \log \left(\frac{a}{b}\right)\right|<|\log (-\lambda)| ; 1^{\alpha}:=1\right) .
\end{aligned}
$$


It is easy to see that setting $a=1$ and $b=c=e$ in (1.8) would lead to Apostol-Euler polynomials defined by (1.4). The case where $a=1$ has been studied by Luo et al. [8].

In Section 2, we introduce the new extension of unified family of Apostol-type polynomials and numbers that are defined in [9]. Also, we determine relations between some results given in [1] [3] [7] [10] [11] and our results. Moreover, we introduce some new identities for polynomials defined in [9]. In Section 3, we give some basic properties of the new unification of Apostol-type polynomials and numbers. Finally in Section 4, we introduce some relationships between the new unification of Apostol-type polynomials and other known polynomials.

\section{Unification of Multiparameter Apostol-Type Polynomials and Numbers}

Definition 2.1. Let $a, b, c \in \mathbb{R}^{+}(a \neq b), n \in \mathbb{N}_{0}$ and $m \in \mathbb{N}$. Then the new unification of Apostol-type polynomials $M_{n}^{[m-1, r]}\left(x ; k ; a, b, c ; \bar{\alpha}_{r}\right)$ are defined, in a suitable neighbourhood of $t=0$ by means of generating function

$$
\begin{aligned}
& F_{\bar{\alpha}_{r}}^{[m-1, r]}=\frac{t^{r k m} 2^{r m(1-k)} c^{x t}}{\prod_{i=0}^{r-1}\left(\alpha_{i} b^{t}-a^{t} \sum_{\ell=0}^{m-1} \frac{t^{\ell}}{\ell !}\right)}=\sum_{n=0}^{\infty} M_{n}^{[m-1, r]}\left(x ; k ; a, b, c ; \bar{\alpha}_{r}\right) \frac{t^{n}}{n !} \\
& \left(\left|t \log \left(\frac{b}{a}\right)\right|<2 \pi \text { when } m=1 \text { and } \alpha_{i}=1 ;\left|t \log \left(\frac{b}{a}\right)\right|<\left|\log \left(\alpha_{i}\right)\right| \text { when } m=1 \text { and } \alpha_{i} \neq 1 ; \forall i=0,1, \cdots, r-1\right),
\end{aligned}
$$

where $k \in N_{0} ; r \in \mathbb{C} ; \bar{\alpha}_{r}=\left(\alpha_{0}, \alpha_{1}, \cdots, \alpha_{r-1}\right)$ is a sequence of complex numbers.

Remark 2.1. If we set $x=0$ in (2.1), then we obtain the new unification of multiparameter Apostol-type numbers, as

$$
M_{n}^{[m-1, r]}\left(0 ; k ; a, b, c ; \bar{\alpha}_{r}\right)=M_{n}^{[m-1, r]}\left(k ; a, b, c ; \bar{\alpha}_{r}\right) .
$$

The generating function in (2.1) gives many types of polynomials as special cases, for example, see Table 1 .

Remark 2.2. From NO. 13 in Table 1 and ([9], Table 1), we can obtain the polynomials and the numbers given in [12]-[16].

\section{Some Basic Properties for the Polynomial $M_{n}^{[m-1, r]}\left(x ; k ; a, b, c ; \bar{\alpha}_{r}\right)$}

Theorem 3.1. Let $a, b, c \in \mathbb{R}^{+}(a \neq b)$ and $x \in R$. Then

$$
\begin{aligned}
& M_{n}^{[m-1, r]}\left(x+y ; k ; a, b, c ; \bar{\alpha}_{r}\right)=\sum_{l=0}^{n}\left(\begin{array}{l}
n \\
l
\end{array}\right) x^{n-l}(\ln c)^{n-l} M_{l}^{[m-1, r]}\left(y ; k ; a, b, c ; \bar{\alpha}_{r}\right) . \\
& M_{n}^{[m-1, r]}\left(x+r ; k ; a, b, c ; \bar{\alpha}_{r}\right)=M_{n}^{[m-1, r]}\left(x ; k ; \frac{a}{c}, \frac{b}{c}, c ; \bar{\alpha}_{r}\right) .
\end{aligned}
$$

Proof. For the first equation, from (2.1)

$$
\sum_{n=0}^{\infty} M_{n}^{[m-1, r]}\left(x+y ; k ; a, b, c ; \bar{\alpha}_{r}\right) \frac{t^{n}}{n !}=\frac{t^{r k m} 2^{r m(1-k)} c^{x t}}{\prod_{i=0}^{r-1}\left(\alpha_{i} b^{t}-a^{t} \sum_{\ell=0}^{m-1} \frac{t^{\ell}}{\ell !}\right)} c^{y t}=\sum_{j=0}^{\infty} \frac{(t y \ln c)^{j}}{j !} \sum_{l=0}^{\infty} M_{l}^{[m-1, r]}\left(x ; k ; a, b, c ; \bar{\alpha}_{r}\right) \frac{t^{l}}{l !},
$$

using Cauchy product rule, we can easily obtain (3.1).

For the second Equation (3.2), from (2.1)

$$
\sum_{n=0}^{\infty} M_{n}^{[m-1, r]}\left(x+r ; k ; a, b, c ; \bar{\alpha}_{r}\right) \frac{t^{n}}{n !}=\frac{t^{r k m} 2^{r m(1-k)}}{\prod_{i=0}^{r-1}\left(\alpha_{i}\left(\frac{b}{c}\right)^{t}-\left(\frac{a}{c}\right)^{t} \sum_{\ell=0}^{m-1} \frac{t^{\ell}}{\ell !}\right)} c^{x t}=\sum_{n=0}^{\infty} M_{n}^{[m-1, r]}\left(x ; k ; \frac{a}{c}, \frac{b}{c}, c ; \bar{\alpha}_{r}\right) \frac{t^{n}}{n !} .
$$


Table 1. Special cases.

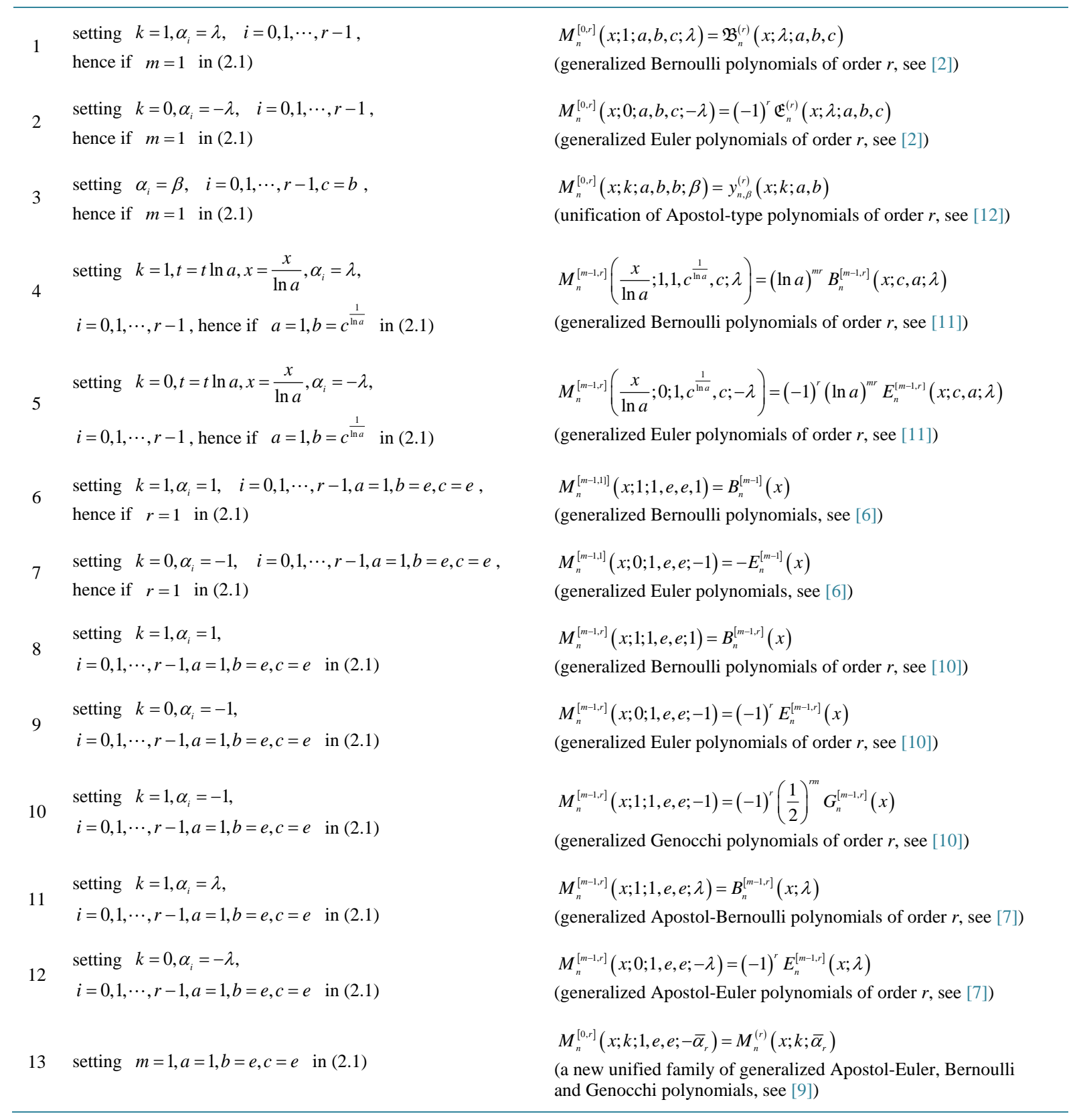

Equating the coefficient of $\frac{t^{n}}{n !}$ on both sides, yields (3.2).

Corollary 3.1. If $y=0$ in (3.1), we have

$$
\begin{aligned}
& M_{n}^{[m-1, r]}\left(x ; k ; a, b, c ; \bar{\alpha}_{r}\right)=\sum_{\ell=0}^{n}\left(\begin{array}{l}
n \\
\ell
\end{array}\right) x^{n-\ell}(\ln c)^{n-\ell} M_{\ell}^{[m-1, r]}\left(k ; a, b, c ; \bar{\alpha}_{r}\right) \\
& =\sum_{\ell=0}^{n}\left(\begin{array}{c}
n \\
n-\ell
\end{array}\right) x^{\ell}(\ln c)^{\ell} M_{n-\ell}^{[m-1, r]}\left(k ; a, b, c ; \bar{\alpha}_{r}\right) .
\end{aligned}
$$

Theorem 3.2. The following identity holds true, when $m=1$ and $\alpha_{i} \neq 0$ in (2.1) $\forall i=0,1, \cdots, r-1$ 


$$
M_{n}^{[0, r]}\left(r-x ; k ; a, b, c ; \bar{\alpha}_{r}\right)=\frac{(-1)^{r(1-k)+n}}{\prod_{i=0}^{r-1} \alpha_{i}} \sum_{m=0}^{n}\left(\begin{array}{c}
n \\
m
\end{array}\right)\left(r \ln \left(\frac{a b}{c}\right)\right)^{n-m} M_{m}^{[0, r]}\left(x ; k ; a, b, c ; \frac{1}{\bar{\alpha}_{r}}\right) .
$$

Proof. From (2.1)

$$
\begin{aligned}
& \sum_{n=0}^{\infty} M_{n}^{[0, r]}\left(r-x ; k ; a, b, c ; \bar{\alpha}_{r}\right) \frac{t^{n}}{n !} \\
= & \frac{t^{r k} 2^{r(1-k)} c^{(r-x) t}}{\prod_{i=0}^{r-1}\left(\alpha_{i} b^{t}-a^{t}\right)}=\frac{(-1)^{r(1-k)}}{\left(b^{t} a^{t}\right)^{r} \prod_{j=0}^{r-1} \alpha_{j}} \frac{(-t)^{r k} 2^{r(1-k)} c^{-\chi t}\left(\frac{b^{-t}}{\alpha_{i}}-a^{-t}\right)}{c^{r t}} \\
= & \frac{(-1)^{r(1-k)}}{\prod_{j=0}^{r-1} \alpha_{j}}\left(\frac{b a}{c}\right)^{-r t} \sum_{m=0}^{\infty} M_{m}^{[0, r]}\left(x ; k ; a, b, c ; \frac{1}{\bar{\alpha}_{r}}\right) \frac{(-t)^{m}}{m !} \\
= & \frac{(-1)^{r(1-k)}}{\prod_{j=0}^{r-1} \alpha_{j}} \sum_{\ell=0}^{\infty} \frac{\left(r \ln \left(\frac{a b}{c}\right)\right)^{\ell}}{\ell !}(-t)^{\ell} \sum_{m=0}^{\infty} M_{m}^{[0, r]}\left(x ; k ; a, b, c ; \frac{1}{\bar{\alpha}_{r}}\right) \frac{(-t)^{m}}{m !} .
\end{aligned}
$$

Hence, we can easily obtain (3.5).

Remark 3.1. If we put $\alpha_{i}=\beta, i=0,1, \cdots, r-1, c=b$ and $r=v$ in (3.5), then it gives [[12], Equation (34)],

$$
M_{n}^{[0, v]}(v-x ; k ; a, b, b ; \beta)=\frac{(-1)^{v(1-k)+n}}{(\beta)^{v}} \sum_{m=0}^{n}\left(\begin{array}{c}
n \\
m
\end{array}\right)(v \ln a)^{n-m} M_{m}^{[0, v]}\left(x ; k ; a, b, b ; \beta^{-1}\right),
$$

where $M_{m}^{[0, v]}\left(x ; k ; a, b, b ; \beta^{-1}\right)$ is the unification of the Apostol-type polynomials.

Theorem 3.3. The unification of Apostol-type numbers satisfy

$$
M_{n}^{[m-1, r]}\left(k ; a, b, c ; \bar{\alpha}_{r}\right)=\sum_{l=0}^{n}\left(\begin{array}{l}
n \\
l
\end{array}\right) M_{l}^{[m-1, \ell]}\left(k ; a, b, c ; \bar{\alpha}_{\ell}\right) M_{n-l}^{[m-1, r-\ell]}\left(k ; a, b, c ; \bar{\alpha}_{r-\ell}\right) .
$$

Proof. When $x=0$ in (2.1), we have

$$
\begin{aligned}
& \sum_{n=0}^{\infty} M_{n}^{[m-1, r]}\left(k ; a, b, c ; \bar{\alpha}_{r}\right) \frac{t^{n}}{n !} \\
& =\frac{t^{r k m} 2^{r m(1-k)}}{\prod_{i=0}^{r-1}\left(\alpha_{i} b^{t}-a^{t} \sum_{\ell=0}^{m-1} \frac{t^{\ell}}{\ell !}\right)}=\frac{t^{\ell k m} 2^{\ell m(1-k)}}{\prod_{i=0}^{\ell-1}\left(\alpha_{i} b^{t}-a^{t} \sum_{\ell=0}^{m-1} \frac{t^{\ell}}{\ell !}\right)} \frac{t^{(r-\ell) k m} 2^{(r-\ell) m(1-k)}}{\prod_{i=\ell}^{r-1}\left(\alpha_{i} b^{t}-a^{t} \sum_{\ell=0}^{m-1} \frac{t^{\ell}}{\ell !}\right)} \\
& =\sum_{\ell_{1}=0}^{\infty} M_{\ell_{1}}^{[m-1, \ell]}\left(k ; a, b, c ; \bar{\alpha}_{\ell}\right) \frac{t^{\ell_{1}}}{\ell_{1} !} \sum_{\ell_{2}=0}^{\infty} M_{\ell_{2}}^{[m-1, r-\ell]}\left(k ; a, b, c ; \bar{\alpha}_{r-\ell}\right) \frac{t^{\ell_{2}}}{\ell_{2} !} .
\end{aligned}
$$

Using Cauchy product rule, we obtain (3.6).

Theorem 3.4. The following relationship holds true

$$
\sum_{k_{1}+k_{2}+\cdots+k_{\ell}=n} \prod_{i=1}^{\ell} \frac{M_{k_{i}}^{\left[m-1, r_{i}\right]}\left(x_{i} ; k ; a, b, c ; \bar{\alpha}_{r_{i}}\right)}{k_{1} ! k_{2} ! \cdots k_{\ell} !}=\frac{1}{n !} M_{n}^{[m-1, r \mid r]}\left(|x| ; k ; a, b, c ; \bar{\alpha}_{|r|}\right),
$$

where $|\boldsymbol{r}|=r_{1}+r_{2}+\cdots+r_{\ell}$ and $|\boldsymbol{x}|=x_{1}+x_{2}+\cdots+x_{\ell}$ and $\bar{\alpha}_{r_{i}}=\left(\alpha_{\sum_{j=1}^{i-1} r_{j}}, \alpha_{\sum_{j=1}^{i-1} r_{j}+1}, \cdots, \alpha_{\sum_{j=1}^{i} r_{j}-1}\right), \quad i=\{1,2, \cdots, \ell\}$.

Proof. Starting with (2.1), we get 


$$
\begin{aligned}
& \sum_{n=0}^{\infty}\left(M_{n}^{[m-1, r \mid r]}\left(|x| ; k ; a, b, c ; \bar{\alpha}_{|r|}\right)\right) \frac{t^{n}}{n !} \\
& =\frac{t^{|r| k m} 2^{|r| m(1-k)} c^{|x| t}}{\prod_{i=0}^{|r|-1}\left(\alpha_{i} b^{t}-a^{t} \sum_{\ell=0}^{m-1} \frac{t^{\ell}}{\ell !}\right)}
\end{aligned}
$$

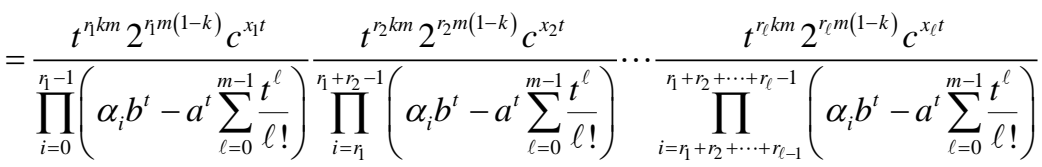

$$
\begin{aligned}
& =\sum_{k_{1}=0}^{\infty} M_{k_{1}}^{\left[m-1, r_{1}\right]}\left(x_{1} ; k ; a, b, c ; \bar{\alpha}_{r_{1}}\right) \frac{t^{k_{1}}}{k_{1} !} \sum_{k_{2}=0}^{\infty} M_{k_{2}}^{\left[m-1, r_{2}\right]}\left(x_{2} ; k ; a, b, c ; \bar{\alpha}_{r_{2}}\right) \frac{t^{k_{2}}}{k_{2} !} \ldots \sum_{k_{\ell}=0}^{\infty} M_{k_{1}}^{\left[m-1, r_{\ell}\right]}\left(x_{\ell} ; k ; a, b, c ; \bar{\alpha}_{r_{\ell}}\right) \frac{t^{k_{\ell}}}{k_{\ell} !}
\end{aligned}
$$

Using Cauchy product rule on the right hand side of the last equation and equating the coefficients of $t^{n}$ on both sides, yields (3.7).

Using No. 13 in Table 1, we obtain Nörlund's results, see [17] and Carlitz's generalizations, see [18] by our approach in Theorem 3.5 and Theorem 3.6 as follows

Theorem 3.5. For $\left(\bar{\alpha}_{r}\right)^{n}=\left(\alpha_{0}^{n}, \alpha_{1}^{n}, \cdots, \alpha_{r-1}^{n}\right)$, we have

$$
\begin{aligned}
& \prod_{i=1}^{r} \sum_{s_{i}=0}^{n-1}\left(\alpha_{i-1}\right)^{s_{i}} M_{\ell}^{[0, r]}\left(x+\frac{\sum_{i=1}^{r} s_{i}}{n} ; k ; 1, e, e ;\left(\bar{\alpha}_{r}\right)^{n}\right)=n^{r k-\ell} M_{\ell}^{[0, r]}\left(n x+; k ; 1, e, e ; \bar{\alpha}_{r}\right) . \\
& \prod_{i=1}^{r} \sum_{s_{i}=0}^{n-1}\left(\alpha_{i-1}\right)^{s_{i}} M_{r+\ell}^{[0, r]}\left(x+\frac{\sum_{i=0}^{r} s_{i}}{n} ; k ; 1, e, e ;\left(\bar{\alpha}_{r}\right)^{n}\right)=n^{r(k-1)-\ell} \frac{(\ell+r) !}{\ell !} M_{\ell}^{[0, r]}\left(n x+; k-1 ; 1, e, e ; \bar{\alpha}_{r}\right) .
\end{aligned}
$$

Proof. For the first equation and starting with (2.1), we get

$$
\begin{aligned}
& \sum_{\ell=0}^{\infty} \frac{(n t)^{\ell}}{\ell !} \prod_{i=1}^{r} \sum_{s_{i}=0}^{n-1}\left(\alpha_{i-1}\right)^{s_{i}} M_{\ell}^{[0, r]}\left(x+\frac{\sum_{i=1}^{r} s_{i}}{n} ; k ; 1, e, e ;\left(\bar{\alpha}_{r}\right)^{n}\right) \\
& =\frac{(n t)^{r k} 2^{r(1-k)} \mathrm{e}^{n x t}}{\prod_{i=0}^{r-1} \alpha_{i}^{n} \mathrm{e}^{n t}-1} \prod_{i=1}^{r} \sum_{s_{i}=0}^{n-1}\left(\alpha_{i-1} \mathrm{e}^{t}\right)^{s_{i}}=\frac{(n t)^{r k} 2^{r(1-k)} \mathrm{e}^{(n x) t}}{\prod_{i=0}^{r-1} \alpha_{i} e^{t}-1}=n^{r k} \sum_{\ell=0}^{\infty} M_{\ell}^{[0, r]}\left(n x ; k ; 1, e, e ; \bar{\alpha}_{r}\right) \frac{t^{\ell}}{\ell !} .
\end{aligned}
$$

Equating the coefficients of $t^{\ell}$ on both sides, yields (3.8).

For the second equation and starting with (2.1), we get

$$
\begin{aligned}
& \sum_{\ell=0}^{\infty} \frac{(n t)^{\ell}}{\ell !} \prod_{i=1}^{r} \sum_{s_{i}=0}^{n-1}\left(\alpha_{i-1}\right)^{s_{i}} M_{\ell}^{[0, r]}\left(x+\frac{\sum_{i=1}^{r} s_{i}}{n} ; k ; 1, e, e ;\left(\bar{\alpha}_{r}\right)^{n}\right) \\
& =\frac{n^{r k} t^{r} 2^{-r}(t)^{r(k-1)} 2^{(2-k)} \mathrm{e}^{n x t}}{\prod_{i=0}^{r-1} \alpha_{i}^{n} \mathrm{e}^{n t}-1} \prod_{i=1}^{r} \sum_{s_{i}=0}^{n-1}\left(\alpha_{i-1} \mathrm{e}^{t}\right)^{s_{i}}=\frac{n^{r k} t^{r} 2^{-r}(t)^{r(k-1)} 2^{r(2-k)} \mathrm{e}^{n x t}}{\prod_{i=0}^{r-1} \alpha_{i} \mathrm{e}^{t}-1},
\end{aligned}
$$

then, we have 


$$
\begin{aligned}
& \sum_{\ell=0}^{\infty} \frac{n^{\ell+r} \ell !}{(\ell+r) !} \frac{t^{\ell}}{\ell !} \prod_{i=1}^{r} \sum_{s_{i}=0}^{n-1}\left(\alpha_{i-1}\right)^{s_{i}} M_{r+\ell}^{[0, r]}\left(x+\frac{\sum_{i=1}^{r} s_{i}}{n} ; k ; 1, e, e ;\left(\bar{\alpha}_{r}\right)^{n}\right) \\
& =n^{r k} 2^{-r} \sum_{\ell=0}^{\infty} M_{\ell}^{[0, r]}\left(n x ; k-1 ; 1, e, e ; \bar{\alpha}_{r}\right) \frac{t^{\ell}}{\ell !} .
\end{aligned}
$$

Equating coefficients of $t^{\ell}$ on both sides, yields (3.9).

Theorem 3.6. For $\left(\overline{\boldsymbol{\alpha}}_{r}\right)^{n}=\left(\alpha_{0}^{n}, \alpha_{1}^{n}, \cdots, \alpha_{r-1}^{n}\right)$ and $\left(\overline{\boldsymbol{\alpha}}_{r}\right)^{m}=\left(\alpha_{0}^{m}, \alpha_{1}^{m}, \cdots, \alpha_{r-1}^{m}\right)$ we have

$$
\begin{aligned}
& n^{\ell} \prod_{i=1}^{r} \sum_{s_{i}=0}^{n-1}\left(\alpha_{i-1}\right)^{m s_{i}} M_{\ell}^{[0, r]}\left(\frac{x}{n}+\frac{\left(\sum_{i=1}^{r} s_{i}\right) m}{n} ; k ; 1, e, e ;\left(\bar{\alpha}_{r}\right)^{n}\right) \\
& =m^{-r k+\ell} n^{r k} \prod_{i=1}^{r} \sum_{p_{i}=0}^{m-1}\left(\alpha_{i-1}\right)^{n p_{i}} M_{\ell}^{[0, r]}\left(\frac{x}{m}+\frac{\left(\sum_{i=1}^{r} p_{i}\right) n}{m} ; k ; 1, e, e ;\left(\bar{\alpha}_{r}\right)^{m}\right) . \\
& n^{\ell+r} \prod_{i=1}^{r} \sum_{s_{i}=0}^{n-1}\left(\alpha_{i-1}\right)^{m s_{i}} M_{\ell+r}^{[0, r]}\left(\frac{x}{n}+\frac{\left(\sum_{i=1}^{r} s_{i}\right) m}{n} ; k ; 1, e, e ;\left(\bar{\alpha}_{r}\right)^{n}\right) \\
& =\frac{m^{-r(k-1)+\ell} n^{r k}}{2^{r}} \prod_{i=1}^{r} \sum_{p_{i}=0}^{m-1}\left(\alpha_{i-1}\right)^{n p_{i}} M_{\ell}^{[0, r]}\left(\frac{x}{m}+\frac{\left(\sum_{i=1}^{r} p_{i}\right) n}{m} ; k-1 ; 1, e, e ;\left(\bar{\alpha}_{r}\right)^{m}\right) .
\end{aligned}
$$

Proof. For the first equation and starting with (2.1), we get

$$
\begin{aligned}
& \sum_{\ell=0}^{\infty} \frac{(n t)^{\ell}}{\ell !} \prod_{i=1}^{r} \sum_{s_{i}=0}^{n-1}\left(\alpha_{i-1}\right)^{m s_{i}} M_{\ell}^{[0, r]}\left(\frac{x}{n}+\frac{\left(\sum_{i=1}^{r} s_{i}\right) m}{n} ; k ; 1, e, e ;\left(\bar{\alpha}_{r}\right)^{n}\right) \\
= & \frac{n^{r k} 2^{r(1-k)} t^{r k} \mathrm{e}^{x t} \prod_{i=0}^{r-1}\left(\alpha_{i} \mathrm{e}^{n m t}-1\right)}{\prod_{i=0}^{r-1}\left(\alpha_{i}^{n} \mathrm{e}^{n t}-1\right) \prod_{i=0}^{r-1}\left(\alpha_{i}^{m} \mathrm{e}^{m t}-1\right)} \\
= & \frac{\left.m^{-r k} n^{r k} 2^{r(1-k)} t^{r k} m^{r k} \mathrm{e}^{\left.\frac{x}{m}+\frac{\left(\sum_{i=1}^{r} p_{i}\right)}{m}\right)}\right)_{m t}^{r-1}\left(\alpha_{i}^{m} \mathrm{e}^{m t}-1\right)}{\prod_{i=0}^{r}} \sum_{p_{i}=0}^{m-1}\left(\alpha_{i-1}\right)^{n p_{i}} \\
= & m^{-r k} n^{r k} \sum_{\ell=0}^{\infty}\left(\prod_{i=1}^{r} \sum_{p_{i}=0}^{m-1}\left(\alpha_{i-1}\right)^{n p_{i}} m^{\ell} M_{\ell}^{[0, r]}\left(\frac{x}{m}+\frac{\left(\sum_{i=1}^{r} p_{i}\right) n}{m} ; k ; 1, e, e ;\left(\bar{\alpha}_{r}\right)^{m}\right)\right)
\end{aligned}
$$


Equating the coefficients of $t^{\ell}$ on both sides, yields (3.10).

Also, It is not difficult to prove (3.11).

\section{Some Relations between $M_{n}^{[m-1, r]}\left(x ; k ; a, b, c ; \bar{\alpha}_{r}\right)$ and Other Polynomials and}

\section{Numbers}

In this section, we give some relationships between the polynomials $M_{n}^{[m-1, r]}\left(x ; k ; a, b, c ; \bar{\alpha}_{r}\right)$ and Laguerre polynomials, Jacobi polynomials, Hermite polynomials, generalized Stirling numbers of second kind, Stirling numbers and Bleimann-Butzer-hahn basic.

Theorem 4.1. For $\bar{\alpha}_{r}=\left(\alpha_{0}, \alpha_{1}, \cdots, \alpha_{r}\right) \in \mathbb{C},(x ; \bar{\alpha})_{\ell}=\left(x-\alpha_{0}\right)\left(x-\alpha_{1}\right) \cdots\left(x-\alpha_{\ell-1}\right)$ and $n, j \in \mathbb{N}_{0}$, we have relationship

$$
M_{n}^{[m-1, r]}\left(x ; k ; a, b, c ; \bar{\alpha}_{r}\right)=\sum_{j=0}^{n}(x ; \alpha)_{\underline{j}} \sum_{\ell=j}^{n}\left(\begin{array}{c}
n \\
n-\ell
\end{array}\right)(\ln c)^{\ell} S(\ell, j ; \bar{\alpha}) M_{n-\ell}^{[m-1, r]}\left(k ; a, b, c ; \bar{\alpha}_{r}\right)
$$

between the new unification of Apostol-type polynomials and generalized Stirling numbers of second kind, see [19].

Proof. Using (3.4) and from definition of generalized Stirling numbers of second kind, we easily obtain (4.1).

Theorem 4.2. For $\bar{\alpha}_{r}=\left(\alpha_{0}, \alpha_{1}, \cdots, \alpha_{r}\right) \in \mathbb{C},(x)_{\ell}=(x)(x-1) \cdots(x-\ell+1)$ and $n, j \in \mathbb{N}_{0}$, we have the relationship

$$
M_{n}^{[m-1, r]}\left(x ; k ; a, b, c ; \bar{\alpha}_{r}\right)=\sum_{j=0}^{n}(x)_{\underline{j}} \sum_{\ell=j}^{n}\left(\begin{array}{c}
n \\
n-\ell
\end{array}\right)(\ln c)^{\ell} S(\ell, j) M_{n-\ell}^{[m-1, r]}\left(k ; a, b, c ; \bar{\alpha}_{r}\right)
$$

between the new unification of Apostol-type polynomials and Stirling numbers of second kind.

Proof. Using (3.4) and from definition of Stirling numbers of second kind (see [20]), we easily obtain (4.2).

Theorem 4.3. The relationship

$$
M_{n}^{[m-1, r]}\left(x ; k ; a, b, c ; \bar{\alpha}_{r}\right)=\sum_{j=0}^{n} \sum_{\ell=j}^{n}(-1)^{j} \ell !\left(\begin{array}{c}
n \\
n-\ell
\end{array}\right)(\ln c)^{\ell}\left(\begin{array}{c}
\ell+\alpha \\
\ell-j
\end{array}\right) L_{j}^{(\alpha)}(x) M_{n-\ell}^{[m-1, r]}\left(k ; a, b, c ; \bar{\alpha}_{r}\right)
$$

holds between the new unification of multiparameter Apostol-type polynomials and generalized Laguerre polynomials (see [7], No. (3), Table 1).

Proof. From (3.4) and substitute

$$
x^{\ell}=\ell ! \sum_{j=0}^{\ell}(-1)^{j}\left(\begin{array}{c}
\ell+\alpha \\
\ell-j
\end{array}\right) L_{j}^{\alpha}(x)
$$

then we get (4.3).

Theorem 4.4. For $(\alpha+\beta+j+1)_{\ell+1}=(\alpha+\beta+j+1)(\alpha+\beta+j+2) \cdots(\alpha+\beta+j+\ell+1)$. The relationship

$$
\begin{aligned}
& M_{n}^{[m-1, r]}\left(x ; k ; a, b, c ; \bar{\alpha}_{r}\right)=\sum_{j=0 \ell=j}^{n} \sum^{n}(-1)^{j} \ell !\left(\begin{array}{c}
n \\
n-\ell
\end{array}\right)(\ln c)^{\ell}\left(\begin{array}{c}
\ell+\alpha \\
\ell-j
\end{array}\right) \frac{\alpha+\beta+2 j+1}{(\alpha+\beta+j+1)_{\ell+1}} \\
& P_{j}^{(\alpha, \beta)}(1-2 x) M_{n-\ell}^{[m-1, r]}\left(k ; a, b, c ; \bar{\alpha}_{r}\right)
\end{aligned}
$$

holds between the new unification of Apostol-type polynomials and Jacobi polynomials (see [21], p. 49, Equation (35)).

Proof. From (3.4) and substitute

$$
x^{\ell}=\ell ! \sum_{j=0}^{\ell}(-1)^{j}\left(\begin{array}{c}
\ell+\alpha \\
\ell-j
\end{array}\right) \frac{\alpha+\beta+2 j+1}{(\alpha+\beta+j+1)_{\ell+1}} P_{j}^{(\alpha, \beta)}(1-2 x),
$$

then we get (4.4).

Theorem 4.5. The relationship 


$$
M_{n}^{[m-1, r]}\left(x ; k ; a, b, c ; \bar{\alpha}_{r}\right)=\sum_{j=0}^{\left[\frac{n}{2}\right]} \sum_{\ell=2 j}^{n} 2^{-\ell}\left(\begin{array}{c}
n \\
n-\ell
\end{array}\right)\left(\begin{array}{c}
\ell \\
2 j
\end{array}\right) \frac{2 j !}{j !}(\ln c)^{\ell} H_{\ell-2 j}(x) M_{n-\ell}^{[m-1, r]}\left(k ; a, b, c ; \bar{\alpha}_{r}\right)
$$

holds between the new unification of Apostol-type polynomials and Hermite polynomials (see [7], No. (1) Table 1).

Proof. From (3.4) and substitute

$$
\left.x^{\ell}=2^{-\ell} \sum_{j=0}^{\left[\frac{\ell}{2}\right.}\right]\left(\begin{array}{c}
\ell \\
2 j
\end{array}\right) \frac{2 j !}{j !} H_{\ell-2 j}(x)
$$

then we get (4.5).

Theorem 4.6. When $m=1, a=1, b=e$ and $c=e$ in (9) and for $\bar{\alpha}_{r}=\left(\alpha_{0}, \alpha_{1}, \cdots, \alpha_{r-1}\right)$, ${\overline{\alpha^{*}}}_{r}=\left(\frac{1}{\alpha_{0}}, \frac{1}{\alpha_{1}}, \cdots, \frac{1}{\alpha_{r-1}}\right), \alpha_{i} \neq 0, i=0,1, \cdots, r-1$ and $\overline{\boldsymbol{\beta}}_{m}=\left(\beta_{0}, \beta_{1}, \cdots, \beta_{m-1}\right),{\overline{\boldsymbol{\beta}^{*}}}_{m}=\left(\frac{1}{\beta_{0}}, \frac{1}{\beta_{1}}, \cdots, \frac{1}{\beta_{m-1}}\right)$, $\beta_{i} \neq 0, \quad i=0,1, \cdots, m-1$, we have the following relationship

$$
M_{n}^{(r)}\left(x ; k ; \bar{\alpha}_{r}\right)=\frac{n !}{\prod_{i=0}^{r-1} \alpha_{i}} \sum_{m=r}^{\infty} \frac{2^{(1-k)(r-m)} \prod_{j=0}^{m-1} \beta_{j}}{(n+k(m-1)) !} C\left(m, r ; \bar{\alpha}_{r}^{*} ;{\overline{\beta^{*}}}_{m}\right) M_{n+k(m-r)}^{(m)}\left(x ; k ; \bar{\beta}_{m}\right),
$$

between the new unified family of generalized Apostol-Euler, Bernoulli and Genocchi polynomials, and $C\left(m, r ; \bar{\alpha}_{r}^{*} ; \bar{\beta}_{m}^{*}\right)$ (the generalized Lah numbers) (see [22]).

Proof. From [9], Equation (2.1),

$$
\begin{aligned}
& \sum_{n=0}^{\infty} M_{n}^{(r)}\left(x ; k ; \bar{\alpha}_{r}\right) \frac{t^{n}}{n !} \\
& =\frac{t^{r k} 2^{r(1-k)} \mathrm{e}^{x t}}{\prod_{i=1}^{r-1}\left(\alpha_{i} \mathrm{e}^{t}-1\right)}=\frac{t^{r k} 2^{r(1-k)} \mathrm{e}^{\mathrm{xt}}}{\prod_{i=1}^{r-1} \alpha_{i}} \frac{1}{\left(e^{t} ; \overline{\alpha^{*}}\right)_{\underline{r}}} \\
& =\frac{t^{r k} 2^{r(1-k)} \mathrm{e}^{x t}}{\prod_{i=1}^{r-1} \alpha_{i}} C\left(m, r ; \overline{\alpha^{*}}{ }_{r} ; \overline{\beta^{*}}{ }_{m}\right) \frac{1}{\left(\mathrm{e}^{t} ; \overline{\beta^{*}}\right)_{\underline{m}}} \\
& =\sum_{m=r}^{\infty} \frac{t^{k(r-m)} 2^{(r-m)(1-k)} \prod_{j=1}^{m-1} \beta_{j}}{\prod_{i=1}^{r-1} \alpha_{i}} C\left(m, r ; \overline{\alpha^{*}}{ }_{r} ;{\overline{\beta^{*}}}_{m}\right) \frac{t^{m k} 2^{m(1-k)} \mathrm{e}^{x t}}{\left(\mathrm{e}^{t} ; \bar{\beta}^{*}\right)_{m}} \\
& =\sum_{n=0}^{\infty}\left(\sum_{m=r}^{\infty} \frac{n ! 2^{(1-k)(r-m)} \prod_{j=0}^{m-1} \beta_{j}}{(n+k(m-1)) ! \prod_{i=0}^{r-1} \alpha_{i}} C\left(m, r ; \overline{\alpha^{*}} r ;{\overline{\beta^{*}}}_{m}\right) M_{n+k(m-r)}^{(m)}\left(x ; k ; \bar{\beta}_{m}\right)\right) \frac{t^{n}}{n !} .
\end{aligned}
$$

Equating the coefficients of $t^{n}$ on both sides, yields (4.6).

Using No. 13 in Table 1 (see [9]) and the definition of the unified Bernstein and Bleimann-Butzer-Hahn basis (see [23]),

$$
\left(\frac{2^{1-k} x^{k} t^{k}}{(1+a x)^{k}}\right)^{m} \frac{1}{m k !} \mathrm{e}^{t\left(\frac{1+b x}{1+a x}\right)}=\sum_{n=0}^{\infty} p_{n}^{(a, b)}(x ; k, m) \frac{t^{n}}{n !}
$$


where $k, m \in \mathbb{Z}^{+}, a, b \in \mathbb{R}, \quad t \in \mathbb{C}$, we obtain the following theorem.

Theorem 4.7. For $\alpha_{i} \neq 0, i=0,1, \cdots, r-1$, we have relationship

$$
P_{n}^{(a, b)}(x ; k, r)=\frac{\prod_{i=0}^{r-1} \alpha_{i}}{r k !}\left(\frac{x}{1+a x}\right)^{r k} \sum_{j=0}^{r} s\left(r, j ; \frac{1}{\bar{\alpha}_{r}}\right) \sum_{\ell=0}^{n} j^{n-\ell}\left(\begin{array}{l}
n \\
\ell
\end{array}\right) M_{\ell}^{(r)}\left(\frac{1+b x}{1+a x} ; k ; \bar{\alpha}_{r}\right)
$$

between the unified Bernstein and Bleimann-Butzer-Hahn basis, the new unified family of generalized Apostol-Bernoulli, Euler and Genocchi polynomials (see [9]) and generalized Stirling numbers of first kind (see [19]).

Proof. From (2.1) and (4.7) and with some elementary calculation, we easily obtain (4.8).

\section{References}

[1] Srivastava, H.M., Garg, M. and Choudhary, S. (2010) A New Generalization of the Bernoulli and Related Polynomials, Russian. Journal of Mathematical Physics, 17, 251-261. http://dx.doi.org/10.1134/S1061920810020093

[2] Srivastava, H.M., Garg, M. and Choudhary, S. (2011) Some New Families of Generalized Euler and Genocchi Polynomials. Taiwanese Journal of Mathematics, 15, 283-305.

[3] Srivastava, H.M. and Pintér, Á. (2004) Remarks on Some Relationships between the Bernoulli and Euler Polynomials. Applied Mathematics Letters, 17, 375-380. http://dx.doi.org/10.1016/S0893-9659(04)90077-8

[4] Luo, Q.-M. and Srivastava, H.M. (2005) Some Generalizations of the Apostol Bernoulli and Apostol Euler Polynomials. Journal of Mathematical Analysis and Applications, 308, 290-302. http://dx.doi.org/10.1016/j.jmaa.2005.01.020

[5] Luo, Q.-M. (2006) Apostol-Euler Polynomials of Higher Order and Gaussian Hypergeometric Functions. Taiwanese Journal of Mathematics, 10, 917-925.

[6] Natalini, P. and Bernardini, A. (2003) A Generalization of the Bernoulli Polynomials. Journal of Applied Mathematics, 153-163. http://dx.doi.org/10.1155/s1110757x03204101

[7] Tremblay, R., Gaboury, S. and Fugère, B.-J. (2011) A New Class of Generalized Apostol-Bernoulli Polynomials and Some Analogues of the Srivastava-Pintér Addition Theorem. Applied Mathematics Letters, 24, 1888-1893. http://dx.doi.org/10.1016/j.aml.2011.05.012

[8] Luo, Q.-M., Guo, B.-N., Qui, F. and Debnath, L. (2003) Generalizations of Bernoulli Numbers and Polynomials. International Journal of Mathematics and Mathematical Sciences, 59, 3769-3776. http://dx.doi.org/10.1155/S0161171203112070

[9] El-Desouky, B.S. and Gomaa, R.S. (2014) A New Unified Family of Generalized Apostol-Euler, Bernoulli and Genocchi Polynomials. Applied Mathematics and Computation, 247, 695-702. http://dx.doi.org/10.1016/j.amc.2014.09.002

[10] Kurt, B. (2010) A Further Generalization of Bernoulli Polynomials and on 2D-Bernoulli Polynomials $B_{n}^{2}(x, y)$. Applied Mathematical Sciences, 47, 2315-2322.

[11] Kurt, B. (2013) Some Relationships between the Generalized Apostol-Bernoulli and Apostol-Euler Polynomials. Turkish Journal of Analysis and Number Theory, 1, 54-58.

[12] Ozden, H. and Simsek, Y. (2014) Modification and Unification of the Apostol-Type Numbers and Polynomials and Their Applications. Applied Mathematics and Computation, 235, 338-351. http://dx.doi.org/10.1016/j.amc.2014.03.004

[13] Apostol, T.M. (1951) On the Lerch Zeta Function. Pacific Journal of Mathematics, 1, 161-167. http://dx.doi.org/10.2140/pjm.1951.1.161

[14] Dere, R., Simsek, Y. and Srivastava, H.M. (2013) A Unified Presentation of Three Families of Generalized Apostol Type Polynomials Based upon the Theory of the Umbral Calculus and the Umbral Algebra. Journal of Number Theory, 133, 3245-3263. http://dx.doi.org/10.1016/j.jnt.2013.03.004

[15] Karande, B.K. and Thakare, N.K. (1975) On the Unification of Bernoulli and Euler Polynomials. Indian Journal of Pure and Applied Mathematics, 6, 98-107.

[16] Luo, Q.-M. (2004) On the Apostol Bernoulli Polynomials. Central European Journal of Mathematics, 2, 509-515. http://dx.doi.org/10.2478/BF02475959

[17] Nörlund, N.E. (1924) Vörlesunge über differezerechnung. Springer-Verlag, Berlin. http://dx.doi.org/10.1007/978-3-642-50824-0

[18] Carlitz, L. (1962) Some Generalized Multiplication Formulae for the Bernoulli Polynomials and Related Functions. Monatshefte für Mathematik, 66, 1-8. 
[19] Comtet, L. (1972) Nombers de Stirling generaux et fonctions symetriques. Comptes Rendus de l'Académie des Sciences (Series A), 275, 747-750.

[20] Gould, H.W. (1960) Stirling Number Representation Problems. Proceedings of the American Mathematical Society, 11, 447-451. http://dx.doi.org/10.1090/S0002-9939-1960-0114767-8

[21] Srivastava, H.M. and Choi, J. (2001) Series Associated with the Zeta and Related Functions. Kluwer Academic, Dordrecht. http://dx.doi.org/10.1007/978-94-015-9672-5

[22] Charalambides, C.A. (2005) Generalized Stirling and Lah Numbers. In: Charalambides, C.A., Ed., Combinatorial Methods in Discrete Distributions, John Wiley \& Sons, Inc., Hoboken, 121-158.

[23] Özarslan, M.A. and Bozer, M. (2013) Unified Bernstein and Bleimann-Butzer-Hahn Basis and Its Properties. Advances in Difference Equations, 2013, 55. http://dx.doi.org/10.1186/1687-1847-2013-55 